\title{
The complete mitochondrial genomes of five longicorn beetles (Coleoptera: Cerambycidae) and phylogenetic relationships within Cerambycidae
}

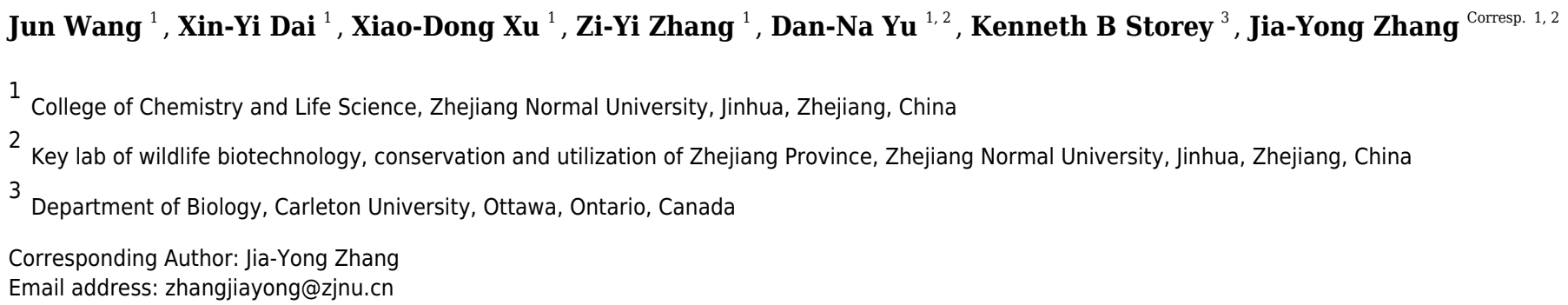

Cerambycidae is one of the most diversified groups within Coleoptera and includes nearly 35,000 known species. The relationships at the subfamily level within Cerambycidae have not been convincingly demonstrated and the gene rearrangement of mitochondrial genomes in Cerambycidae remains unclear due to the low numbers of sequenced mitogenomes. In the present study, we determined five complete mitogenomes of Cerambycidae and investigated the phylogenetic relationship among the subfamilies of Cerambycidae based on mitogenomes. The mitogenomic arrangement of all five species was identical to the ancestral Cerambycidae type without gene rearrangement. Remarkably, however, two large intergenic spacers were detected in the mitogenome of Pterolophia sp. ZJY-2019. The origins of these intergenic spacers could be explained by the slipped-strand mispairing and duplication/random loss models. A conserved motif was found between trnS2 and nad1 gene, which was proposed to be a binding site of a transcription termination peptide. Also, tandem repeat units were identified in the A+T-rich region of all five mitogenomes. The monophyly of Lamiinae and Prioninae was strongly supported by both MrBayes and RAXML analyses based on nucleotide datasets, whereas the Cerambycinae and Lepturinae were recovered as non-monophyletic. 
1 The complete mitochondrial genomes of five longicorn beetles

2 (Coleoptera: Cerambycidae) and phylogenetic relationships within

3 Cerambycidae

4 Jun Wang ${ }^{1}$, Xin-Yi Dai ${ }^{1}$, Xiao-Dong Xu${ }^{1}$, Zi-Yi Zhang ${ }^{1}$, Dan-Na Yu ${ }^{1,2}$, Kenneth B. Storey ${ }^{3}$, Jia-

5 Yong Zhang ${ }^{1,2}$

6

$7{ }^{1}$ College of Chemistry and Life Science, Zhejiang Normal University, Jinhua, 321004, Zhejiang

8 Province, China

$9{ }^{2}$ Key lab of wildlife biotechnology, conservation and utilization of Zhejiang Province, Zhejiang

10 Normal University, Jinhua, 321004, Zhejiang Province, China

$11{ }^{3}$ Department of Biology, Carleton University, Ottawa, Ontario, K1S5B6, Canada

13 Corresponding author: JY Zhang: zhang3599533@163.com or zhangjiayong@zjnu.cn

15 Other authors:

16 J Wang: 17388152521@163.com

17 XY Dai: kkr_xy@163.com

18 XD Xu:ki01110015@163.com

19 ZY Zhang: cixi55@126.com

KB Storey: kenneth.storey@carleton.ca

DN Yu: ydn@zjnu.cn 


\section{ABSTRACT}

27 Cerambycidae is one of the most diversified groups within Coleoptera and includes nearly 35,000 known species. The relationships at the subfamily level within Cerambycidae have not been convincingly demonstrated and the gene rearrangement of mitochondrial genomes in Cerambycidae remains unclear due to the low numbers of sequenced mitogenomes. In the present study, we determined five complete mitogenomes of Cerambycidae and investigated the phylogenetic relationship among the subfamilies of Cerambycidae based on mitogenomes. The mitogenomic arrangement of all five species was identical to the ancestral Cerambycidae type without gene rearrangement. Remarkably, however, two large intergenic spacers were detected in the mitogenome of Pterolophia sp. ZJY-2019. The origins of these intergenic spacers could be explained by the slipped-strand mispairing and duplication/random loss models. A conserved motif was found between trnS2 and nadl gene, which was proposed to be a binding site of a transcription termination peptide. Also, tandem repeat units were identified in the A+T-rich region of all five mitogenomes. The monophyly of Lamiinae and Prioninae was strongly supported by both MrBayes and RAxML analyses based on nucleotide datasets, whereas the

41 Cerambycinae and Lepturinae were recovered as non-monophyletic.

Keywords: Mitochondrial genome, Cerambycidae, Intergenic spacer, Phylogenetic relationship 
47

48

49

50

51

52

\section{INTRODUCTION}

Coleoptera (Hexapoda: Insecta) are a highly diverse group of insects consisting of about 360,000 known species of beetles that account for almost $40 \%$ of all described insect species (Lawrence \& Newton, 1982; Hunt et al., 2007). Cerambycidae (longicorn beetles) is one of the species-rich families of Coleoptera and is a group of phytophagous insects with over 4000 genera and 35,000 species in the world (Monné ML, Monné \& Mermudes, 2009; Sama et al., 2010). Longicorn beetles are morphologically and ecologically diverse, and have significant effects on almost all terrestrial ecosystems (Ponomarenko \& Prokin, 2015). Nevertheless, owing to their remarkable species richness, variable morphological features and sparse gene data, the resolution of the phylogeny of longicorn beetles has turned out to be a difficult challenge (Bologna et al., 2008; Zhang et al., 2018c). Cerambycidae s. s. (sensu stricto) has usually been divided into eight subfamilies: Lamiinae, Cerambycinae, Lepturinae, Prioninae, Dorcasominae, Parandrinae, Spondylidinae and Necydalinae (Svacha, Wang \& Chen, 1997) whereas Cerambycidae s. $l$. (sensu lato) was considered to consist of Cerambycidae s. s., Disteniidae, Oxypeltidae and Vesperidae (Napp,1994; Reid, 1995; Svacha, Wang \& Chen, 1997). Even if the number and definition of Cerambycidae gradually stabilizes, the relationships at the subfamily level remained unclear.

The mitochondrial genome is widely considered to be an informative molecular marker for species identification, molecular evolution, and comparative genomic research (Moritz, Dowling \& Brown, 1987; Boore, 1999) due to its maternal inheritance and high evolutionary rate properties (Avise et al., 1987). In the last few years, studies of animal mitogenomes have grown rapidly in number and approximately 40,000 mitogenome sequences have now been published in the NCBI database (Tan et al., 2017). By contrast, a mere 18 sequenced mitogenomes of Cerambycidae have been reported, among them being eight mitogenomes belonging to the subfamily Lamiinae, four mitogenomes of the subfamily Cerambycinae, three mitogenomes of the subfamily Prioninae, and three mitogenomes of the subfamily Lepturinae (Kim et al., 2009; 
Chiu et al., 2016; Fang et al., 2016; Guo et al., 2016; Li et al., 2016a; Li et al., 2016b; Wang et al., 2016; Lim et al., 2017; Liu et al., 2017; Song et al., 2017; Liu et al., 2018; Que et al., 2019; Wang et al., 2019). These few mitogenomes seriously restrict the capacity for phylogenetic analyses and phylogeography of the Cerambycidae.

The gene organization of the known mitogenomes of Coleoptera, especially the arrangements of protein-coding genes, are mostly in accordance with those of ancestral insects (Timmermans, Martijn \& Vogler, 2012). Nevertheless, recent evidence suggested that gene rearrangements had occurred in the tRNA of Mordella atrata (Coleoptera: Mordellidae) and Naupactus xanthographus (Coleoptera: Curculionidae) (Song et al., 2010). In addition to these, recombination in the control region was observed in Phrixtothrix hirtus (Coleoptera: Phengodidae) and Teslasena femoralis (Coleoptera: Elateridae) (Amaral et al., 2016). The mitogenome structure was originally found with no introns, sparse intergenic spacers and no overlapping genes (Ojala, Montoya \& Attardi, 1981). Nevertheless, large non-coding regions (except the A+T-rich region) in mitogenomes have been observed within beetles, including a 1724-bp long intergenic spacer region in Pyrocoelia rufa (Coleoptera: Lampyridae), a 494-bp region in Hycleus chodschenticus (Coleoptera: Meloidae) and two large intergenic spacers of more than 30 bp in Hycleus species (Bae et al., 2004; Yuan et al., 2016; Haddad et al., 2018). Previously reported tandem repeat units or an additional origin of replication were identified among large intergenic regions (Dotson \& Beard, 2001; Rodovalho et al., 2014).

The phylogenetic relationships within Cerambycidae have yet to be fully resolved due to a lack of adequately convincing taxon sampling, and the monophyly of subfamilies within Cerambycidae need further discuss (Haddad et al., 2018; Kim et al., 2018). With the aim to discuss the monophyly of subfamilies of Cerambycidae and gene arrangements of the mitogenome, complete mitogenomes of the five longicorn beetle species were determined. We also described the structural and compositional features of the newly sequenced mitogenomes and analyzed the intergenic spacers to explain the possible evolutionary mechanisms.

\section{MATERIALS AND METHODS}




\section{Sampling collection and DNA extraction}

Five longicorn beetle specimens (Oberea yaoshana, Thermistis croccocincta, Blepephaeus succinctor, Nortia carinicollis, Pterolophia sp. ZJY-2019) were captured from Jinxiu, Guangxi Zhuang Autonomous Region, China and were stored at $-40{ }^{\circ} \mathrm{C}$ in the lab of JY Zhang (College of Chemistry and Life Science, Zhejiang Normal University). The specimens were identified by Dr. JY Zhang based on morphology. Total genomic DNA was extracted from the thorax muscle using Ezup Column Animal Genomic DNA Purification Kit (Sangon Biotech Company, Shanghai, China).

\section{PCR amplification and sequencing}

In order to obtain the entire mitogenome of samples, we used eleven universal primer pairs to amplify eleven adjacent and overlapping fragments (Simon et al., 2006; Zhang et al., 2008; Zhang et al., 2018a, 2018b). Then specific primers were designed from the initial overlapping fragments using Primer Premier 5.0 (Premier Biosoft International, Palo Alto, CA). A total of 45 pairs of primers were used in the present study to amplify and sequence the remaining gaps (Table S1). The cycling conditions and reaction volume of PCR amplifications were as in Cheng et al. (2016) and Gao et al. (2018). All PCR products were sequenced by Sangon Biotech Company (Shanghai, China).

\section{Mitogenome annotation and sequence analyses}

Manual proofreading and assembling of contiguous and overlapping sequences used DNASTAR Package v.6.0 (Burland, 2000). We annotated the tRNA genes by MITOS (freely available at http://mitos.bioinf.uni-leipzig.de/index.py) (Bernt et al., 2013). Two rRNA genes and the A+T-rich region were identified using the Clustal W in Mega 7.0 (Kumar, Stecher \& Tamura, 2016) based on alignments of homologous sequences from other species of Cerambycidae available in the GenBank (Kim et al., 2009; Fang et al., 2016; Lim et al., 2017). The nucleotide sequences of the 13 protein-coding genes (PCGs) were translated into amino acids based on the invertebrate mitogenome genetic code (Cameron, 2014). We used Mega 7.0 (Kumar, Stecher \& Tamura, 2016) to find the open reading frames of the 13 PCGs and calculate 
129

130

131

132

133

134

135

136

137

138

139

140

141

142

143

144

145

146

147

148

149

150

151

152

153

154

155

156

AT content along with codon usage for the five newly sequenced mitogenomes. Circular mitogenome maps were generated by CG View server V 1.0 (Grant \& Stothard, 2008). Composition skew analysis was calculated on the basis of the formula AT-skew $=(\mathrm{A}-\mathrm{T}) /(\mathrm{A}+$ $\mathrm{T})$ and GC-skew $=(\mathrm{G}-\mathrm{C}) /(\mathrm{G}+\mathrm{C})($ Perna \& Kocher, 1995). Tandem Repeat Finder V 4.07 (http://tandem.bu.edu/trf/trf.html) (Benson, 1999) was used to find tandem repetitive sequences.

\section{Phylogenetic analyses}

For the purpose of reconstructing the phylogenetic relationships of Cerambycidae, a nucleotide dataset (13P26) of the 13 protein-coding genes of 26 complete mitogenomes was used (Table 1) according to the methods of Zhang et al. (2019), this included the 5 newly determined sequences and 18 published complete mitogenomes of Cerambycidae (Kim et al., 2009; Chiu et al., 2016; Fang et al., 2016; Guo et al., 2016; Li et al., 2016a; Li et al., 2016b; Wang et al., 2016; Lim et al., 2017; Liu et al., 2017; Song et al., 2017; Liu et al., 2018; Que et al., 2019; Wang et al., 2019). Three species of Galerucinae, Paleosepharia posticata, Diabrotica barberi and Diabrotica virgifera served as the out-groups (Coates \& Brad, 2014; Wang \& Tang, 2017). To verify whether the lack of samples affects the relationships among the Cerambycidae, we reconstructed Cerambycidae phylogeny based on the nucleotide data (12P38) of 12 PCGs (omitting the $n a d 2$ gene) from 38 complete or nearly complete mitogenomes (Table 1). These include all species of the 13P26 dataset, 8 directly submitted partial mitogenomes of Cerambycidae, one mitogenome of Necydalinae, two mitogenomes of Vesperidae and one mitogenome of Disteniidae (Nie et al., 2017). Each of the 13 protein-coding genes in 13P26 dataset or 12 protein-coding genes in 12P38 dataset was aligned using Clustal $\mathrm{W}$ in the program Mega 7.0 (Kumar, Stecher \& Tamura, 2016). Conserved regions were identified by the program Gblock 0.91b (Castresana, 2000). Protein-coding genes were partitioned a priori by codon position. Accodrding to the analyses methods of Zhang et al. (2008), Ma et al. (2015) and Cheng et al. (2016), we excluded the third codon positions because of the saturated third codon positions and obtained a 12P38 dataset with 5584 nucleotide sites and 13P26 dataset with 6960 nucleotide sites. So $12 \mathrm{P} 38$ dataset with 24 partitions and $13 \mathrm{P} 26$ dataset with 26 partitions were 
157

158

159

160

161

162

163

164

165

166

167

168

169

170

171

172

173

174

175

176

177

178

179

180

181

182

183

used. The optimal partitioning scheme and best-fitting models were selected by the program PartitionFinder 1.1.1 (Lanfear et al., 2012) based on the Bayesian information criterion (BIC) (Table 2 and Table 3). Bayesian Inference (BI) and Maximum likelihood (ML) methods were used for phylogenetic analyses. BI analyses were carried out in MrBayes 3.2 (Ronquist et al., 2012) with the model of GTR + I + G. The runs were set for 10 million generations with sampling every 1,000 generations. The first $25 \%$ of generations were removed as burn-in and the average standard deviation of split frequencies in Bayesian was below 0.01. ML analyses were performed by RAxML 8.2.0 with the best-fitting model of GTRGAMMAI. Branch support values were inferred from a rapid bootstrap method applied with 1,000 replications (Stamatakis, 2014).

\section{RESULTS AND DISCUSSION}

\section{Mitogenome organization and composition}

In this study, the complete mitogenomes of five species of the subfamilies Cerambycinae and Lamiinae (O. yaoshana, T. croccocincta, B. succinctor, N. carinicollis, Pterolophia sp. ZJY2019) were determined. Structures of the five newly sequenced entire mitogenomes are shown in Fig. S1-S5. The lengths of the five mitogenomes were basically within the range of the published Cerambycidae species in the GenBank database, covering sizes between 15,503 bp in $T$. croccocincta to 16,063 bp in Pterolophia sp. ZJY-2019. Every mitogenome of the five species possessed similar compositional profiles and featured the typical gene arrangement and orientation that have been hypothesized for most coleopteran insects (Wolstenholme, 1992; Boore, Lavrov \& Brown, 1998), with the trnW-trnC-trnY triplet (Table S2-S6). Twenty-three genes were coded on the majority strand (J-strand), with the remaining fourteen genes coded on the minority strand (N-strand) (Fig. S1-S5). The nucleotide composition of the five longicorn beetle mitogenomes was strongly biased towards A and T, which made up $73.2 \%$ (N. carinicollis) to $79.1 \%$ (O. yaoshana) of the base pairs. A comparison of AT-skew and GC-skew showed that the AT skew of all mitogenomes was positive and the GC-skew was negative (Table 4). 


\section{Protein-coding genes and codon usages}

The orientations of the 13 the PCGs of the five longicorn beetles were identical to most coleopteran species (Table S2-S6). Conventional initiation codons were assigned to the majority of the PCGs, except for nadl, which started with TTG in all five beetles. Most putative protein sequences showed typical stop codons (TAA/TAG), but the nad4 and nad5 genes of $O$. yaoshana, T. croccocincta, B. succinctor used a single T residue as the terminal codon. The cox 1 and cox 2 genes of O. yaoshana, T. croccocincta and Pterolophia sp. ZJY-2019 also used a single T residue as the terminal codon. Functional terminal codons can be produced by partial terminal codons in polycistronic transcription cleavage and polyadenylation processes (Anderson et al., 1981; Ojala, Montoya \& Attardi, 1981; Du et al., 2016). The relative synonymous codon usage (RSCU) of the five Cerambycidae mitochondrial genomes was calculated (Fig. 1, Table S7). The results showed an over-utilization of A or T nucleotides in the third codon position as compared to other synonymous codons, this is normally considered to be caused by genome bias, optimum choice of tRNA usage or the benefit of DNA repair (Chai \& Du, 2012; Ma et al., 2015).

Comparative analyses also indicated that the major customarily utilized codons and the codon usage patterns of the five samples were conservative. For instance, each of the five mitogenomes possessed UUA (Leu), AUU (Ile), UUU (Phe), and AUA (Met) as the most frequently used codons. All codons contained A or T nucleotides, indicating that the strong AT mutation bias obviously influenced the codon usage (Powell \& Moriyama, 1997; Rao et al., 2011). Furthermore, the codons rich in AT encoded the most abundant amino acids, e.g. Leu (15.6-16.4\%), indicating that the AT bias also influences the amino acid constituents of the proteins encoded by the mitochondrial genes (Foster et al., 1997; Min \& Hickey, 2007).

\section{Ribosomal RNAs and transfer RNAs}

The two expected rRNAs (16S rRNA and 12S rRNA) were found in the mitochondrial genomes of all five longicorn beetles. The $16 \mathrm{~S}$ rRNA gene was situated between $\operatorname{trn} L$ and $\operatorname{trn} V$ whereas the $12 \mathrm{~S}$ rRNA gene was between $\operatorname{trn} V$ and the A+T-rich region. Due to the impossibility 
211 of faultless determination by DNA sequence alone, the terminus of the rRNA genes in 212 coleopteran mitogenomes has been presumed to stretch to the border of the flanking genes 213 (Boore, 2001). Therefore, the 16S rRNA was presumed to fill the blank between trnL and trnV 214 whereas the border between $12 \mathrm{~S}$ rRNA and the putative A+T-rich region was defined based on 215 alignments of homologous sequences of known longicorn beetles (Boore \& Brown, 2000). The 216 sizes of $16 \mathrm{~S}$ rRNA in the five beetle mitogenomes varied from $1261 \mathrm{bp}$ for $N$. carinicollis to $2171283 \mathrm{bp}$ for O. yaoshana, and the sizes of 12S rRNA ranged between $759 \mathrm{bp}$ for Pterolophia sp. 218 ZJY-2019 to 787 bp for $T$. croccocincta. These fit within the lengths detected in other coleopteran mitogenomes. The $\mathrm{A}+\mathrm{T}$ content of the rRNA genes was the highest $(81.7 \%)$ in the Pterolophia sp. ZJY-2019 mitogenome and the lowest in the N. carinicollis mitogenome (75.7\%). The AT-skew of 16S rRNA and 12S rRNA showed great positivity, whereas the GC-skew was somewhat negative (Table 4), which indicated the occurrence of less As and Cs than Ts and Gs (Eyrewaker, 1997).

The 22 typical tRNAs were detected in all five species like other published longicorn beetles. All the anticodons were also highly conserved compared to other beetle species. Twentytwo tRNAs excluding trnS1 displayed the classic clover-leaf secondary structure, whereas trnS1 lacked the dihydrouridine (DHU) arm and formed a simple loop (Fig. S6). Nevertheless, this abnormal tRNA has proven to be functional, although somewhat less effective than conventional tRNAs (Steinberg \& Cedergren, 1994; Hanada et al., 2001; Stewart \& Beckenbach, 2003). Another unusual feature was the use of TCT as the trnS1 anticodon in Cerambycidae, whereas most arthropods use a GCT anticodon in trnS1. In many other coleopteran mitogenomes the trnS1 anticodon (TCT) can also be observed (Friedrich \& Muqim, 2003; Bae et al., 2004). Mismatched pairs also exist in stems of tRNAs. For example, the mismatched pairs U-G existed in the DHU stem of $\operatorname{trn} Y$ and $\operatorname{trn} Q$; U-U existed in the TYC stem of $\operatorname{trn} C$ and in the anticodon stem of trnL1; G-U existed in acceptor stem of $\operatorname{trnC}$. It has been verified that mismatched pairs can be revised via editing processes or may symbolize abnormal pairings (Negrisolo, Babbucci \& Patarnello, 2011). 
239

240

241

242

243

244

245

246

247

248

249

250

251

252

253

254

255

256

257

258

259

260

261

262

263

264

265

266

\section{A+T-rich region}

A large non-coding region between 12S rRNA and trnI, ranging between $861 \mathrm{bp}$ for $O$. yaoshana to 1137 bp for Pterolophia sp. ZJY-2019, was found in the mitogenomes of the five beetles. Owing to the high AT content levels of the overall mitogenome, this non-coding element was defined as the $\mathrm{A}+\mathrm{T}$-rich region. It has been verified that the $\mathrm{A}+\mathrm{T}$-rich region harbors the origin sites and essential regulatory elements for transcription and replication (Wolstenholme, 1992; Taanman, 1999; Yukuhiro et al., 2002; Saito, Tamura \& Aotsuka, 2005). The sequence of this region is relatively conserved owing to its high $\mathrm{A}+\mathrm{T}$ content, and thus it is impossible to use as a molecular marker (Zhang \& Hewitt, 1997). The existence of tandem repeats in the mitochondrial A+T-rich region has been observed in many coleopteran species. Some studies such as that conducted by Sheffield et al. (2008) have shown that the A+T-rich region of Trachypachus holmbergi (Coleoptera: Trachypachidae) possessed 21 similar copies of tandem repeats consisting of a 58-bp fragment. The A+T-rich region of Priasilpha obscura (Coleoptera: Phloeostichidae) is known to possess 6 tandem repeats of a 132-bp fragment and Psacothea hilaris (Coleoptera: Cerambycidae) possesses 7 identical copies of a 57 bp tandem repeat (Kim et al., 2009). In the present study, we found tandem repetitive sequences in all five newly sequenced mitogenomes. The mitogenomes of $T$. croccocincta and B. succinctor contained three copies of tandem repetitive sequences with lengths of 19 and $43 \mathrm{bp}$, respectively. Four tandem repeats of a 19-bp fragment were found in the mitogenome of Pterolophia sp. ZJY-2019, whereas two tandem repeats of a 25-bp fragment existed in $N$. carinicollis. The tandem repeats generally exhibited high $\mathrm{A}+\mathrm{T}$ contents. Moreover, two poly-T stretches were detected in the mitogenome of $N$. carinicollis: one stretch was 16 bp in length (position: 14,880-14,895) near the 12S rRNA gene and the other stretch was 17-bp in length (position: 15,283-15,299). Previous studies have confirmed that the two poly-T stretches were structural signals for the recognition of proteins that performed a role in replication initiation (Andrews, Kubacka \& Chinnery, 1999).

\section{Intergenic regions}


The mitogenomes of $O$. yaoshana, T. croccocincta, and $N$. carinicollis contain 6, 7, 9 non-

268

269

270

271

272

273

274

275

276

277

278

279

280

281

282

283

284

285

286

287

288

289

290

291

292

293

coding intergenic spacer sequences, with total lengths of $28 \mathrm{bp}, 28 \mathrm{bp}$, and $31 \mathrm{bp}$, respectively, whereas $B$. succinctor has 8 non-coding intergenic spacer sequences of $52 \mathrm{bp}$ in total length. Unexpectedly, a total of 354-bp of intergenic spacer, whose elements ranged from 1 to 184 bp in length was found in the mitogenome of Pterolophia sp. ZJY-2019. The sequences are divided into 9 regions, containing two large intergenic spacers. The largest one is $184 \mathrm{bp}$ long situating between $\operatorname{trn} C$ and $\operatorname{trn} Y$, and the other is $157 \mathrm{bp}$ long situated between $\operatorname{trn} S 2$ and nad1 (Table S6). Consequently, the total length of the mitogenome of Pterolophia sp. ZJY-2019 is longer than that of other longicorn beetle species. The longer mitogenome length is due to the existence of its extended large intergenic spacers not the A+T-rich region. Previously reported tandem repeat units or additional origins of replication have been identified within this region (Dotson \& Beard, 2001; Rodovalho et al., 2014). Proven by the lack of introns, rare intergenic spacers, defective terminal codons and overlapping fragments, mitogenomes characteristically show exceptional compactness of organization (Ojala, Montoya \& Attardi, 1981). Nevertheless, according to Yuan et al. (2016) and Haddad et al. (2018), large non-coding regions (except the A+T-rich region) in mitochondrial genomes were observed in Pyrocoelia rufa (Coleoptera: Lampyridae) and some Hycleus species (Coleoptera: Meloidae). Coincidentally, a 5 bp consensus motif (TACTA) exists in the intergenic regions situated between trnS2 and nadl of all five species studied here. This pentanucleotide motif is conserved across coleopteran lineages (Kim et al., 2009; Liu et al., 2018), similar to the findings that Evania appendigaster (Hymenoptera: Evaniidae) possessed a 6 bp motif 'THACWW' and Chilo suppressalis (Lepidoptera: Pyralidae) possessed a 7 bp motif 'ATACTAA', respectively (Wei et al., 2010; Gong et al., 2018).

In the mitogenome of Pterolophia sp. ZJY-2019, the large intergenic region was situated between trnS2 and nad1, which included two copies of a 22 bp long consensus sequence (TTACTAAATTTAATTAACTAAA) in both ends of the intergenic region. The formation of an intergenic region may be explained by slipped-strand mispairing (Levinson \& Gutman, 1987; Du 
294

295

296

297

298

299

300

301

302

303

304

305

306

307

308

309

310

311

312

313

314

315

316

317

318

319

320

321

et al., 2017). Based on this theory, mispairing occurred during replication of DNA strands, and what followed next was misaligned reassociation and then replication or repair was caused by insertions of several repeat units. The resulting tandem repeat underwent random loss and/or point mutation, with only the repeat units in both extremities remaining (Fig. 2a). However, a tandem repeat was not found in the intergenic region located between $\operatorname{trn} C$ and $\operatorname{trn} Y$ of Pterolophia sp. ZJY-2019. We conjectured that some errors in DNA replication can lead to tandem duplication in tRNA clusters of trn $W$-trnC-trnY, followed by the random loss of partial duplicated genes, and leading to the large intergenic region formed by the residues (Fig. 2b). In addition, Hua et al. (2008) suggested that the duplication-random loss model caused the rearrangements in Hemiptera. Du et al. (2017) also suggested that the duplication-random loss model was an evolutionary ancient mechanism in Coleoptera, which led to the random loss of nucleotides.

Consequently, compared to the original tRNAs, the residual intergenic region was not conserved. According to $\mathrm{Du}$ et al. (2017), four species of Hycleus genera harbored similar location and sequence of non-coding regions, which indicated that the region may serve as a latent symbol to distinguish Hycleus from the other genera. Thus, we speculated the large intergenic region of Pterolophia sp. ZJY-2019 may be a molecular feature in Pterolophia, though we were unable to adequately confirm it owing to the lack of enough samples.

\section{Phylogenetic analyses}

The phylogenetic relationships were reconstructed based on the nucleotide data (13P26) with BI and ML methods (Fig. 3). BI and ML phylogenetic analyses yielded a similar topology except for the position of Lepturinae, which was in the sister group of (Cerambycinae + Prioninae) with high values in BI, but supported as the basal group of Cerambycidae in ML analyses. The BI tree indicated that Cerambycidae split into 2 major groups (0.73): a clade of (Lepturinae $+($ Cerambycinae + Prioninae $))$ and a clade of Lamiinae. The monophyly of Lamiinae, Lepturinae and Prioninae was supported by both BI and ML analyses, whereas the monophyly of Cerambycinae was not recovered. Within the subfamily Lamiinae, the clade of 
322 (Lamiinae + (Batocera lineolata + Thyestilla gebleri $)$ ) was supported. However, Liu et al. (2018)

323 favoured T. gebleri as the basal position of Lamiinae with a high value, and B. lineolata and

324 Apriona swainsoni were reliably recovered as a sister group. Our results concurred with the

325 suggestion that $B$. lineolata was closely related to $A$. swainsoni, rather than $T$. gebleri. The

326 results also placed Pterolophia sp. ZJY-2019 as a sister group of all remaining Lamiinae.

327 Moreover, our results suggested that O. yaoshana clustered with Trachypachus holmbergi, as a

328 sister group of $T$. gebleri. For the relationship within Cerambycinae, M. raddei, A. oenochrous

329 and Obrium sp. NS-2015 were gathered into one clade and most closely related to the subfamily

330 Prioninae rather than the remaining Cerambycinae, consistent with the morphological and 331 molecular analyses in previous reports (Liu et al., 2018).

332 The results from the BI trees of the nucleotide dataset showed that Lepturinae cluster with 333 the clade (Cerambycinae + Prioninae) with a high support value (Fig. 3). However, in the ML 334 tree, a close relationship between Lamiinae and (Cerambycinae + Prioninae) was supported with $335100 \%$ posterior probabilities (Fig. 3). The relationship between Cerambycinae and Prioninae is 336 not currently understood in great detail. Prioninae were traditionally considered basal in 337 Cerambycidae by morphology (Hatch, 1956; Svacha, Wang \& Chen, 1997; Farrell,1998). In 338 addition, Hunt et al. (2007) and Haddad et al. (2018) pointed out that Prioninae could be placed 339 at the basal position of Cerambycidae based on molecular phylogenetic studies. However, in BI 340 and ML analyses of the 13P26 dataset Prioninae clustered into Cerambycinae, which was 341 consistent with the phylogenetic position of Prioninae recovered by Raje, Ferris \& Holland 342 (2016).

343 The most controversial point in our results was in Cerambycinae (Fig. 3), which was 344 represented by five different genera and rendered non-monphyletic in Prioninae. However, 345 Cerambycinae was not supported as monophyletic based on molecular by Liu et al. (2018) and 346 Haddad et al. (2018), but was recovered in other molecular studies (Lim et al., 2017; Liu et al., 347 2017).

348 To further discuss the monophyly of subfamilies within Cerambycidae, more samples were 
349

350

351

352

353

354

355

356

357

358

359

360

361

362

363

364

365

366

367

368

369

370

371

372

373

374

375

needed to confirm and rebuild the phylogenetic relationship of Cerambycidae using 12 proteincoding genes. The phylogenetic relationships were reconstructed based on the nucleotide data (12P38) with BI and ML methods (Fig. 4). Prioninae still clustered into Cerambycinae in BI and ML analyses of the $12 \mathrm{P} 38$ dataset, which agreed with the phylogenetic position of Prioninae recovered using the $13 \mathrm{P} 26$ dataset. In $\mathrm{BI}$ and $\mathrm{ML}$ analyses, all trees recovered the monophyly of Lamiinae (although the relationships within Lamiinae were different). The Lamiinae formed a sister group to a clade comprising Disteniidae, Prioninae, Cerambycinae and Vesperidae. The clade of Lepturinae and Necydalinae was a sister to the remaining species of Cerambycidae $s$. $l$. . In addition, BI and ML analyses recovered the monophyly of Prioninae including Callipogon relictus, Dorysthenes paradoxus and Aegosoma sinicum, as proposed by Wang et al. (2019). However, BI and ML results did not support the monophyly of Cerambycinae with respect to Prioninae and Spiniphilus spinicornis (Vesperidae). It has been well accepted that Necydalinae and Lepturinae have a close relationship. The monophyly of Lepturinae was recovered in both BI and ML analyses of the 13P26 dataset. However, BI and ML trees from the 12P38 dataset returned a paraphyletic Lepturinae, due to a sister relationship between Necydalis ulmi (Necydalinae) and Brachyta interrogationis (Fig. 4).

Previous studies recognized S. spinicornis as a species of Vesperinae in Cerambycidae (Napp, 1994). Nevertheless, subsequent studies considered it to belong to the subfamily Philinae of Vesperidae (Svacha, Wang \& Chen, 1997; Lin \& Bi, 2011; Nie et al., 2017). Further phylogenetic studies put S. spinicornis in the fairly controversial placements (Bi \& Lin, 2015; Liu et al., 2018). In addition to our results, a recent molecular study also indicated a similar relationship (Liu et al., 2018).

\section{CONCLUSION}

In this study, we present five completely sequenced mitogenomes of Cerambycidae. The five longicorn beetle species shared similar gene organization with the insects previously reported. The gene sequences and composition of the mitogenomes were relatively conservative with no rearrangements, duplications or deletions. Two large intergenic spacers existed in 
Pterolophia sp. ZJY-2019. The duplication/random loss model and slipped-strand mispairing may explain the existence of these regions. The phylogenetic results inferred from mitogenomes

378 supported the monophyly of Lamiinae and Prioninae in BI and ML analyses, whereas the 379 Cerambycinae and Lepturinae were recovered as non-monophyletic. Although data collected 380 thus far could not resolve the phylogenetic relationships within Cerambycidae, this study will 381 increase the richness of the Cerambycidae genome information and assist in phylogenetic, 382 molecular systematics and evolutionary studies of Cerambycidae. 


\section{References}

Amaral DT, Mitani Y, Ohmiya Y, Viviani VR. 2016. Organization and comparative analysis of the mitochondrial genomes of Bioluminescent Elateroidea (Coleoptera: Polyphaga). Gene 586(2):254-262. DOI 10.1016/j.gene.2016.04.009.

Anderson S, Bankier AT, Barrell BG, Bruijin MHL, Droujn ARJ, Eperon IC, Nierlich DP, Roe BA, Sanger F, Schreier PH. 1981. Sequence and organization of the human mitochondrial genome. Nature 290(5806):457-465. DOI 10.1038/290457a0.

Andrews RM, Kubacka I, Chinnery PF. 1999. Reanalysis and revision of the Cambridge reference sequence for human mitochondrial DNA. Nature Genetics 23(2):147. DOI 10.1038/13779.

Avise JC, Arnold J, Ball RM, Bermingham E, Lamb T, Neigel JE, Reeb CA, Saunders NC. 1987. Intraspecific phylogeography: the mitochondrial DNA bridge between population genetics and systematics. Annual Review of Ecology and Systematics 18(1):489-522. DOI 10.1146/annurev.es.18.110187.002421.

Bae JS, Kim I, Sohn HD, Jin BR. 2004. The mitochondrial genome of the firefly, Pyrocoelia rufa: complete DNA sequence, genome organization, and phylogenetic analysis with other insects. Molecular Phylogenetics and Evolution 32(3):978-985. DOI 10.1016/j.ympev.2004.03.009.

Benson G. 1999. Tandem repeats finder: a program to analyze DNA sequences. Nucleic Acids Research 27(2):573580. DOI 10.1093/nar/27.2.573.

Bernt M, Donath A, Jühling F, Externbrink F, Florentz C, Fritzsch G, Pütz J, Middendorf M, Stadler PF. 2013. MITOS: improved de novo metazoan mitochondrial genome annotation. Molecular Phylogenetics and Evolution 69(2):313-319. DOI 10.1016/j.ympev.2012.08.023.

Bi W, Lin M. 2015. Discovery of second new species of the genus Spiniphilus Lin \& Bi, and female of Heterophilus scabricollis Pu with its biological notes (Coleoptera: Vesperidae: Philinae: Philini). Zootaxa. 3949(4):575-583. DOI 10.11646/zootaxa.3949.4.7.

Bologna MA, Oliverio M, Pitzalis M, Mariottini P. 2008. Phylogeny and evolutionary history of the blister beetles (Coleoptera, Meloidae). Molecular Phylogenetics and Evolution 48(2):679-693. DOI 10.1016/j.ympev.2008.04.019.

Boore JL, Lavrov DV, BrownWM. 1998. Gene translocation links insects and crustaceans. Nature 392(6677):667668. DOI $10.1038 / 33577$.

Boore JL. 1999. Animal mitochondrial genomes. Nucleic Acids Research 27(8):1767-1780. DOI 10.1093/nar/27.8.1767.

Boore JL, Brown WM. 2000. Mitochondrial genomes of Galathealinum, Helobdella, and Platynereis: sequence and gene arrangement comparisons indicate that Pogonophora is not a Phylum and Annelida and Arthropoda are not sister taxa. Molecular Biology and Evolution 17(1):87-106.

DOI10.1093/oxfordjournals.molbev.a026241.

Boore JL. 2001. Complete mitochondrial genome sequence of the polychaete annelid Platynereis dumerilii. Molecular Biology and Evolution 18(7):1413-1416. DOI 10.1186/1471-2164-5-67.

Burland TG. 2000. DNASTAR's Lasergene sequence analysis software. In: Misener S, Krawetz SA, eds. Bioinformatics Methods and Protocols. Methods in Molecular Biology. Totowa: Humana Press, 71-91.

Cameron SL. 2014. How to sequence and annotate insect mitochondrial genomes for systematic and comparative genomics research. Systematic Entomology 39(3):400-411. DOI 10.1111/syen.12071.

Castresana J. 2000. Selection of conserved blocks from multiple alignments for their use in phylogenetic analysis. 
Molecular Biology and Evolution 17(4):540-552. DOI 10.1093/oxfordjournals.molbev.a026334.

Chai HN, Du YZ. 2012. The complete mitochondrial genome of the pink stem borer, Sesamia inferens, in comparison with four other noctuid moths. International Journal of Molecular Sciences 13(8):10236-10256. DOI 10.3390/ijms130810236.

Cheng XF, Zhang LP, Yu DN, Storey KB, Zhang JY. 2016. The complete mitochondrial genomes of four cockroaches (Insecta: Blattodea) and phylogenetic analyses within cockroaches. Gene 586:115-122. DOI 10.1016/j.gene.2016.03.057.

Chiu WC, Yeh WB, Chen ME, Yang MM. 2016. Complete mitochondrial genome of Aeolesthes oenochrous (Fairmaire) (Coleoptera: Cerambycidae): an endangered and colorful longhorn beetle. Mitochondrial DNA Part A. 27(1):686-687. DOI 10.3109/19401736.2014.913143.

Coates, Brad S. 2014. Assembly and annotation of full mitochondrial genomes for the corn rootworm species, Diabrotica virgifera virgifera and Diabrotica barberi (Insecta: Coleoptera: Chrysomelidae), using next generation sequence data. Gene 542(2):190-197. DOI 10.1016/j.gene.2014.03.035.

Dotson EM, Beard CB. 2001. Sequence and organization of the mitochondrial genome of the Chagas disease vector, Triatoma dimidiata. Insect Molecular Biology 10(3):205-215. DOI 10.1046/j.1365-2583.2001.00258.x.

Du C, He SL, Song XH, Liao Q, Zhang XY, Yue BS. 2016. The complete mitochondrial genome of Epicauta chinensis (Coleoptera: Meloidae) and phylogenetic analysis among coleopteran insects. Gene 578(1):274-280. DOI 10.1016/j.gene.2015.12.036.

Du C, Zhang LF, Lu T, Ma JN, Zeng CJ, Yue BS, Zhang XY. 2017. Mitochondrial genomes of blister beetles (Coleoptera, Meloidae) and two large intergenic spacers in Hycleus genera. BMC Genomics 18(1):698. DOI 10.1186/s12864-017-4102-y.

Eyrewaker A. 1997. Differentiating between selection and mutation bias. Genetics 147(4):1983-1987.

Fang J, Qian L, Xu M, Yang X, Wang B, An Y. 2016. The complete nucleotide sequence of the mitochondrial genome of the Asian longhorn beetle, Anoplophora glabripennis (Coleoptera: Cerambycidae). Mitochondrial DNA Part A 27(5):3299-3300. DOI 10.3109/19401736.2015.1015012.

Farrell BD. 1998. "Inordinate fondness" explained: Why are there so many beetles? Science 281(5376):555-559. DOI 10.1126/science.281.5376.555.

Foster PG, Jermiin LS, Hickey DA. 1997. Nucleotide composition bias affects amino acid content in proteins coded by animal mitochondria. Journal of Molecular Evolution 44(3):282-288. DOI 10.1007/p100006145.

Friedrich M, Muqim N. 2003. Sequence and phylogenetic analysis of the complete mitochondrial genome of the flour beetle Tribolium castanaeum. Molecular Phylogenetics and Evolution 26(3):502-512. DOI 10.1016/s1055-7903(02)00335-4.

Gao XY, Cai YY, Yu DN, Storey KB, Zhang JY. 2018. Characteristics of the complete mitochondrial genome of Suhpalacsa longialata (Neuroptera, Ascalaphidae) and its phylogenetic implications. PeerJ 6:e5914. DOI 10.7717/peerj.5914.

Gong R, Guo X, Ma J, Song X, Shen Y, Geng F, Yue B. 2018. Complete mitochondrial genome of Periplaneta brunnea (Blattodea: Blattidae) and phylogenetic analyses within Blattodea. Journal of Asia-Pacific Entomology 21(3): 885-895. DOI 10.1016/j.aspen.2018.05.006.

Grant JR, Stothard P. 2008. The CG View Server: a comparative genomics tool for circular genomes. Nucleic Acids Research 36(2):181-184. DOI 10.1093/nar/gkn179.

Guo K, Chen J, Xu CQ, Qiao HL, Xu R, Zhao XJ. 2016. The complete mitochondrial genome of the longhorn beetle 
Xylotrechus grayii (Coleoptera: Cerambycidae). Mitochondrial DNA Part A 27(3):2133-2134. DOI 10.3109/19401736.2014.982592.

Haddad S, Shin S, Lemmon AR, Lemmon EM, Svacha P, Farrell B, McKenna DD. 2018. Anchored hybrid enrichment provides new insights into the phylogeny and evolution of longhorned beetles (Cerambycidae). Systematic Entomology 43(1):68-89. DOI 10.1111/syen.12257.

Hanada T, Suzuki T, Yokogawa T, Takemoto-Hori C, Sprinzl M, Watanabe K. 2001. Translation ability of mitochondrial tRNAs ${ }^{\mathrm{Ser}}$ with unusual secondary structures in an in vitro translation system of bovine mitochondria. Genes to Cells 6(12):1019-1030. DOI 10.1046/j.1365-2443.2001.00491.x.

Hatch MH. 1956. The natural classification of the families of Coleoptera. Annals of the Entomological Society of America 49(1):102. DOI 10.1093/aesa/49.1.102.

Hua J, Li M, Dong P, Cui Y, Xie Q, Bu W. 2008. Comparative and phylogenomic studies on the mitochondrial genomes of Pentatomomorpha (Insecta: Hemiptera: Heteroptera). BMC Genomics 9(1): 610. DOI 10.1186/1471-2164-9-610.

Hunt T, Bergsten J, Levkanicova Z, Papadopoulou A, John OS, Wild R, Gómez-Zurita J. 2007. A comprehensive phylogeny of beetles reveals the evolutionary origins of a superradiation. Science 318(5858):1913-1916. DOI 10.1126/science.1146954.

Kim KG, Hong MY, Kim MJ, Im HH, Kim MI, Bae CH. 2009. Complete mitochondrial genome sequence of the yellow-spotted long-horned beetle Psacothea hilaris (Coleoptera: Cerambycidae) and phylogenetic analysis among coleopteran insects. Molecules and Cells 27(4):429-441. DOI 10.1007/s10059-009-0064-5.

Kim S, de Medeiros BA, Byun BK, Lee S, Kang JH, Lee B, Farrell BD. 2018. West meets East: How do rainforest beetles become circum-Pacific? Evolutionary origin of Callipogon relictus and allied species (Cerambycidae: Prioninae) in the new and old worlds. Molecular Phylogenetics and Evolution, 125:163-176. DOI 10.1016/j.ympev.2018.02.019.

Kumar S, Stecher G, Tamura K. 2016. Mega 7: molecular evolutionary genetics analysis version 7.0 for bigger datasets. Molecular Biology and Evolution 33(7):1870-1874. DOI 10.1093/molbev/msw054.

Lanfear R, Calcott B, Ho SYW, Guindon S. 2012. PartitionFinder: combined selection of partitioning schemes and substitution models for phylogenetic analyses. Molecular Biology and Evolution 29(6):1695-1701. DOI 10.1093/molbev/mss020.

Lawrence JF, Newton AF. 1982. Evolution and classification of beetles. Annual Review of Ecology and Systematics 13(1):261-290. DOI 10.1146/annurev.es.13.110182.001401.

Levinson G, Gutman GA. 1987. Slipped-strand mispairing: a major mechanism for DNA sequence evolution. Molecular Biology and Evolution 4(3):203-221. DOI 10.1093/oxfordjournals.molbev.a040442.

Li F, Zhang H, Wang W, Weng H, Meng Z. 2016a. Complete mitochondrial genome of the Japanese pine sawyer, Monochamus alternatus (Coleoptera: Cerambycidae). Mitochondrial DNA Part A 27(2):1144-1145. DOI 10.3109/19401736.2014.936321.

Li W, Yang X, Qian L, An Y, Fang J. 2016b. The complete mitochondrial genome of the citrus long-horned beetle, Anoplophora chinensis (Coleoptera: Cerambycidae). Mitochondrial DNA Part A 27(6): 4665-4667. DOI 10.3109/19401736.2015.1106493.

Lim J, Yi DK, Kim YH, Lee W, Kim S, Kang JH. 2017. Complete mitochondrial genome of Callipogon relictus Semenov (Coleoptera: Cerambycidae): a natural monument and endangered species in Korea. Mitochondrial DNA Part B 2(2):629-631. DOI 10.1080/23802359.2017.1372718. 
Lin M, Bi W. 2011. A new genus and species of the subfamily Philinae (Coleoptera: Vesperidae). Zootaxa 2777(1):54-60. DOI 10.11646/zootaxa.2777.1.4.

Liu JH, Jia PF, Luo T, Wang QM. 2017. Complete mitochondrial genome of white-striped long-horned beetle, Batocera lineolata (Coleoptera: Cerambycidae) by next-generation sequencing and its phylogenetic relationship within superfamily Chrysomeloidea. Mitochondrial DNA Part B 2(2):520-521. DOI 10.1080/23802359.2017.1361797.

Liu YQ, Chen DB, Liu HH, Hu HL, Bian HX, Zhang RS. 2018. The complete mitochondrial genome of the longhorn beetle Dorysthenes Paradoxus (Coleoptera: Cerambycidae: Prionini) and the implication for the phylogenetic relationships of the Cerambycidae species. Journal of Insect Science 18(2). DOI 10.1093/jisesa/iey012.

Ma Y, He K, Yu PP, Cheng XF, Zhang JY. 2015. The complete mitochondrial genomes of three bristletails (Insecta: Archaeognatha): the paraphyly of Machilidae and insights into Archaeognathan phylogeny. PLOS ONE 10:e0117669. DOI 10.1371/journal.pone.0117669.

Ma ZH, Yang XF, Bercsenyi M, Wu JJ, Yu Y, Wei K, Qi XF, Yang RB. 2015. Comparative mitogenomics of the genus Odontobutis (Perciformes: Gobioidei: Odontobutidae) revealed conserved gene rearrangement and high sequence variations. International Journal of Molecular Sciences 16(10):25031-25049. DOI 10.3390/ijms 161025031.

Min XJ, Hickey DA. 2007. DNA asymmetric strand bias affects the amino acid composition of mitochondrial proteins. DNA Research 14(5):201-206. DOI 10.1093/dnares/dsm019.

Monné ML, Monné MA, Mermudes JRM. 2009. Inventário das espécies de Cerambycinae (Insecta, Coleoptera, Cerambycidae) do Parque Nacional do Itatiaia, RJ, Brasil. Biota Neotropica 12(12):40-76. DOI 10.1590/S167606032012000100004.

Moritz C, Dowling TE, Brown WM. 1987. Evolution of animal mitochondrial DNA: relevance for population biology and systematics. Annual Review of Ecology and Systematics 18(1):269-292. DOI 10.2307/2097133.

Napp DS. 1994. Phylogenetic relationships among the subfamilies of Cerambycidae (Coleoptera, Chrysomeloidea). Revista Brasileira de Entomologia 38(2):265-419.

Negrisolo E, Babbucci M, Patarnello T. 2011. The mitochondrial genome of the ascalaphid owlfly Libelloides macaronius, and comparative evolutionary mitochondriomics of neuropterid insects. BMC Genomics 12(1):221. DOI 10.1186/1471-2164-12-221.

Nie R, Lin M, Xue H, Bai M, Yang X. 2017. Complete mitochondrial genome of Spiniphilus spinicornis (Coleoptera: Vesperidae: Philinae) and phylogenetic analysis among Cerambycoidea. Mitochondrial DNA Part A 28(1):145-146. DOI 10.3109/19401736.2015.1111363.

Ojala D, Montoya J, Attardi G. 1981. tRNA punctuation model of RNA processing in human mitochondria. Nature 290(5806):470-474. DOI 10.1038/290470a0.

Perna NT, Kocher TD. 1995. Patterns of nucleotide composition at fourfold degenerate sites of animal mitochondrial genomes. Journal of Molecular Evolution 41(3):353-358. DOI 10.1007/bf01215182.

Ponomarenko AG, Prokin AA. 2015. Review of paleontological data on the evolution of aquatic beetles (Coleoptera). Paleontological Journal 49(13):1383-1412. DOI 10.1134/S0031030115130080.

Powell JR, Moriyama EN. 1997. Evolution of codon usage bias in drosophila. Proceedings of the National Academy of Sciences 94(15):7784-7790. DOI 10.2307/2097133.

Que S, Yu A, Liu P, Jin M, Xie GA. 2019. The complete mitochondrial genome of Apriona swainsoni. 
Mitochondrial DNA Part B 4(1):931-932. DOI 10.1080/23802359.2019.1567284.

Raje KR, Ferris VR, Holland JD. 2016. Phylogenetic signal and potential for invasiveness. Agricultural and Forest Entomology 18(3):260-269. DOI 10.1111/afe.12158.

Rao Y, Wu G, Wang Z, Chai X, Nie Q, Zhang X. 2011. Mutation bias is the driving force of codon usage in the Gallus gallus genome. DNA Research 18(6):499-512. DOI 10.1093/dnares/dsr035.

Reid CAM. 1995. A cladistic analysis of subfamilial relationships in the Chrysomelidae s. l. (Chrysomeloidea). Biology, Phylogeny and Classification of Coleoptera: Papers Celebrating the 80th Birthday of Roy A. Crowson 559-631.

Rodovalho CM, Lyra ML, Ferro M, Jr MB. 2014. The mitochondrial genome of the leaf-cutter ant Atta laevigata: a mitogenome with a large number of intergenic spacers. PLoS ONE 9(5): e97117. DOI 10.1371/journal.pone.0097117.

Ronquist F, Teslenko M, Mark PVD, Ayres DL, Darling A, Höhna S, Larget B, Liu L, Suchard MA, Huelsenbeck JP. 2012. MrBayes 3.2: efficient Bayesian phylogenetic inference and model choice across a large model space. Systematic Biology 61(3):539-542. DOI 10.1093/sysbio/sys029.

Saito S, Tamura K, Aotsuka T. 2005. Replication origin of mitochondrial DNA in insects. Genetics 171(4):16951705. DOI 10.1534/genetics.105.046243.

Sama G, Buse J, Orbach E, Friedman A, Rittner O, Chikatunov V. 2010. A new catalogue of the Cerambycidae (Coleoptera) of Israel with notes on their distribution and host plants. Munis Entomology and Zoology 5(1):155. DOI 10.1007/s12032-010-9513-4.

Sheffield NC, Song H, Cameron SL, Whiting MF. 2008. A comparative analysis of mitochondrial genomes in Coleoptera (Arthropoda: Insecta) and genome descriptions of five new beetles. Molecular Biology and Evolution 25(11):2499-2509. DOI 10.1093/molbev/msn198.

Simon C, Buckley TR, Frati F, Stewart JB, Beckenbach AT. 2006. Incorporating molecular evolution into phylogenetic analysis, and a new compilation of conserved polymerase chain reaction primers for animal mitochondrial DNA. Annual Review of Ecology Evolution and Systematics 37(1):545-579. DOI 10.1146/annurev.ecolsys.37.091305.110018.

Song H, Sheffield NC, Cameron SL, Miller KB, Whiting MF. 2010. When phylogenetic assumptions are violated: base compositional heterogeneity and among-site rate variation in beetle mitochondrial phylogenomics. Systematic Entomology 35(3):429-448. DOI 10.1111/j.1365-3113.2009.00517.x.

Song N, Zhang H, Yin X, Lin A, Zhai Q. 2017. The complete mitochondrial genome sequence from the longicorn beetle Obrium sp. (Coleoptera: Cerambycidae). Mitochondrial DNA Part A 28(3):326-327. DOI 10.3109/19401736.2015.1122766.

Stamatakis A. 2014. RAxML version 8: a tool for phylogenetic analysis and post-analysis of large phylogenies. Bioinformatics 30(9):1312-1313. DOI 10.1093/bioinformatics/btu033.

Steinberg S, Cedergren R. 1994. Structural compensation in atypical mitochondrial tRNAs. Nature Structural Biology 1(8):507-510. DOI 10.1038/nsb0894-507.

Stewart JB, Beckenbach AT. 2003. Phylogenetic and genomic analysis of the complete mitochondrial DNA sequence of the spotted asparagus beetle Crioceris duodecimpunctata. Molecular Phylogenetics and Evolution 26(3):513-526. DOI 10.1016/S1055-7903(02)00421-9.

Svacha P, Wang J, Chen S. 1997. Larval morphology and biology of Philus antennatus and Heterophilus punctulatus, and systematic position of the Philinae (Coleoptera: Cerambycidae: Vesperidae). Annales de la 
Société Entomologique de France 33:323-369.

Taanman JW. 1999. The mitochondrial genome: structure, transcription, translation and replication. Biophysica Acta (BBA)-Bioenergetics 1410(2):103-123. DOI 10.1016/S0005-2728(98)00161-3.

Tan MH, Gan HM, Lee YP, Poore GC, Austin CM. 2017. Digging deeper: new gene order rearrangements and distinct patterns of codons usage in mitochondrial genomes among shrimps from the Axiidea, Gebiidea and Caridea (Crustacea: Decapoda). PeerJ 5:e2982. DOI 10.7717/peerj.2982.

Timmermans, Martijn JTN, Vogler AP. 2012. Phylogenetically informative rearrangements in mitochondrial genomes of Coleoptera, and monophyly of aquatic elateriform beetles (Dryopoidea). Molecular Phylogenetics and Evolution 63(2):299-304. DOI 10.1016/j.ympev.2011.12.021

Wang J, Lan DY, Dai XY, Yu DN, Storey KB, Zhang JY. 2019. The complete mitochondrial genome of Xystrocera globosa (Coleoptera: Cerambycidae) and its phylogeny. Mitochondrial DNA Part B 4(1):1647-1649. DOI: $10.1080 / 23802359.2019 .1605852$.

Wang Q, Tang G. 2017. Genomic and phylogenetic analysis of the complete mitochondrial DNA sequence of walnut leaf pest Paleosepharia posticata (Coleoptera: Chrysomeloidea). Journal of Asia-Pacific Entomology 20(3): 840-853. DOI 10.1016/j.aspen.2017.05.010.

Wang YT, Liu YX, Tong XL, Ren QP, Jiang GF. 2016. The complete mitochondrial genome of the longhorn beetle, Massicus raddei. Mitochondrial DNA Part A 27(1):209-211 DOI 10.3109/19401736.2014.880892.

Wei SJ, Tang P, Zheng LH, Shi M, Chen XX. 2010. The complete mitochondrial genome of Evania appendigaster (Hymenoptera: Evaniidae) has low A+ T content and a long intergenic spacer between atp8 and atp6. Molecular Biology Reports 37(4): 1931-1942. DOI 10.1007/s11033-009-9640-1.

Wolstenholme DR. 1992. Animal mitochondrial DNA: structure and evolution. International Review of Cytology 141:173-216. DOI 10.1016/S0074-7696(08)62066-5.

Yuan M, Zhang Q, Zhang L, Guo Z, Liu Y, Shen Y. 2016. High-level phylogeny of the Coleoptera inferred with mitochondrial genome sequences. Molecular Phylogenetics and Evolution 104:99-111. DOI 10.1016/j.ympev.2016.08.002.

Yukuhiro K, Sezutsu H, Itoh M, Shimizu K, Banno Y. 2002. Significant levels of sequence divergence and gene rearrangements have occurred between the mitochondrial genomes of the wild mulberry silkmoth, Bombyx mandarina, and its close relative, the domesticated silkmoth, Bombyx mori. Molecular Biology and Evolution 19(8):1385-1389. DOI 10.1093/oxfordjournals.molbev.a004200.

Zhang JY, Zhou CF, Gai YH, Song DX, Zhou KY. 2008. The complete mitochondrial genome of Parafronurus youi (Insecta: Ephemeroptera) and phylogenetic position of the Ephemeroptera. Gene 424(1-2):18-24. DOI 10.1016/j.gene.2008.07.037.

Zhang DX, Hewitt GM. 1997. Insect mitochondrial control region: A review of its structure, evolution and usefulness in evolutionary studies. Biochemical Systematics and Ecology 25(2):99-120. DOI 10.1016/s03051978(96)00042-7.

Zhang LP, Cai YY, Yu DN. Storey KB, Zhang JY. 2018a. Gene characteristics of the complete mitochondrial genomes of Paratoxodera polyacantha and Toxodera hauseri (Mantodea: Toxoderidae). PeerJ 6:e4595. DOI 10.7717/peerj.4595.

Zhang LP, Ma Y, Yu DN, Storey KB, Zhang JY. 2019. The mitochondrial genomes of Statilia maculata and S. nemoralis (Mantidae: Mantinae) with different duplications of $\operatorname{trnR}$ genes. International Journal of Biological Macromolecules 121:839-845. DOI 10.1016/j.jibiomac.2018.10.038. 
630 Zhang LP, Yu DN, Storey KB, Cheng HY, Zhang JY. 2018b. Higher tRNA gene duplication in mitogenomes of 631 praying mantises (Dictyoptera, Mantodea) and the phylogeny within Mantodea. International Journal of 632 Biological Macromolecules 111:787-795. DOI 10.1016/j.ijbiomac.2018.01.016.

633 Zhang SQ, Che LH, Li Y, Liang D, Pang H, Ślipiński A, Zhang P. 2018c. Evolutionary history of Coleoptera 634 revealed by extensive sampling of genes and species. Nature Communications 9(1):205. DOI 10.1038/s41467$635 \quad 017-02644-4$. 


\section{Ethics Approval and Consent to Participate}

638 All the samples of longicorn beetles were permitted under the scientific research in China.

\section{Competing interests}

640 The authors declare that they have no competing interests.

\section{Acknowledgments}

642 We are grateful to Wen-Yong Feng for his help in sample collection.

\section{Funding}

644 This research was supported by the Zhejiang provincial Natural Science Foundation (Y18C040006), the 645 National Natural Science Foundation of China (31370042), the College students' Innovation and 646 Entrepreneurship Project in China (No. 201810345043), the College students in Zhejiang Normal University

647 Innovation and Entrepreneurship Plan (2018-317) for the study design, data collection and analyses.

\section{Author Contributions}

649 Conceived and designed the experiments: JW XYD DNY JYZ. Performed the experiments: JW XYD. 650 Analyzed the data: JW XYD XDX ZYZ DNY JYZ. Contributed reagents/materials/analysis tools: JW XYD 651 JYZ DNY. Wrote the paper: JW XYD XDX ZYZ DNY KBS JYZ. All the authors read and approved the final 652 version of the manuscript.

\section{ORCID}

654 Jia-Yong Zhang http://orcid.org/0000-0002-7679-2548

655 Dan-Na Yu http://orcid.org/0000-0002-9870-1926

656 Kenneth B. Storey http://orcid.org/000-0002-7363-1853

657 Jun Wang https://orcid.org/0000-0003-1520-8691

658 XY Dai https://orcid.org/0000-0001-6832-1546

$659 \mathrm{XD} \mathrm{Xu}$ https://orcid.org/0000-0002-3682-9967

660 ZY Zhang https://orcid.org/0000-0001-8159-9104 


\section{Figure legends}

663 Figure 1 The RSCU of five longicorn beetle mitochondrial genomes. Codon families are provided on the $\mathrm{x}$-axis along with the

664 different combinations of synonymous codons that code for that amino acid. RSCU (relative synonymous codon usage) is defined 665 on the $\mathrm{Y}$ axis.

666 Figure 2 Putative mechanisms for formation of the two large intergenic regions (IGRs) that exist in Pterolophia sp. ZJY-

667 2019. (a) The slipped-strand mispairing and random loss model to explain the 157 bp-IGR between trnS2 and nad1. The CS

668 indicates the $18 \mathrm{bp}$ conservative sequence TTACTAAATTTAATTAACTAAA. (b) The duplication/random loss model to 669 explain the $184 \mathrm{bp}-\mathrm{IGR}$ between $\operatorname{trn} C$ and $\operatorname{trn} Y$.

670 Figure 3 Phylogenetic relationships of Cerambycidae in BI and ML analyses. The data includes 23 species of Cerambycidae

671 as the ingroup and three species of Chrysomelidae as the outgroup. The GenBank accession numbers of all species are also 672 shown.

673 Figure 4 Phylogenetic relationships of Cerambycidae in BI and ML analyses. The data includes 35 species of Cerambycidae as the ingroup and three species of Chrysomelidae as the outgroup. The GenBank accession numbers of all species are also 675 shown.

676 Figure S1 Mitochondrial genome maps of $\boldsymbol{O}$. yaoshana. The first circle shows the gene map (PCGs, rRNAs, tRNAs and the

677 AT-rich region) and the genes outside the map are coded on the majority strand (J-strand) whereas the genes inside the map are 678 coded on the minority strand (N-strand). The second circle shows the GC content and the third shows the GC skew. Both GC 679 content and GC skew are plotted as the deviation from the average value of the total sequence.

680 Figure S2 Mitochondrial genome maps of $\boldsymbol{T}$. croccocincta. The first circle shows the gene map (PCGs, rRNAs, tRNAs and the 681 AT-rich region) and the genes outside the map are coded on the majority strand (J-strand) whereas the genes inside the map are coded on the minority strand (N-strand). The second circle shows the GC content and the third shows the GC skew. Both GC content and GC skew are plotted as the deviation from the average value of the total sequence.

684 Figure S3 Mitochondrial genome maps of B. succinctor. The first circle shows the gene map (PCGs, rRNAs, tRNAs and the 685 AT-rich region) and the genes outside the map are coded on the majority strand (J-strand) whereas the genes inside the map are 686 coded on the minority strand (N-strand). The second circle shows the GC content and the third shows the GC skew. Both GC 687 content and GC skew are plotted as the deviation from the average value of the total sequence.

688 Figure S4 Mitochondrial genome maps of $\boldsymbol{N}$. carinicollis. The first circle shows the gene map (PCGs, rRNAs, tRNAs and the 689 AT-rich region) and the genes outside the map are coded on the majority strand (J-strand) whereas the genes inside the map are coded on the minority strand (N-strand). The second circle shows the GC content and the third shows the GC skew. Both GC content and GC skew are plotted as the deviation from the average value of the total sequence.

692 Figure S5 Mitochondrial genome maps of Pterolophia sp. ZJY-2019. The first circle shows the gene map (PCGs, rRNAs, 693 tRNAs and the AT-rich region) and the genes outside the map are coded on the majority strand (J-strand) whereas the genes 694 inside the map are coded on the minority strand (N-strand). The second circle shows the GC content and the third shows the GC

695 skew. Both GC content and GC skew are plotted as the deviation from the average value of the total sequence.

696 Figure S6 Secondary structure of tRNAs (trnI-trn $V$ ) in five newly sequenced mitogenomes.

697 (1): O. yaoshana (2): T. croccocincta (3): B. succinctor (4): N. carinicollis (5): Pterolophia sp. ZJY-2019

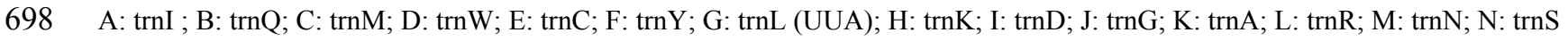
699 (AGN); O: trnE; P: trnF; Q: $\operatorname{trnH}$; R: trnT; S: trnP; T: $\operatorname{trnS}(\mathrm{UCN}) ; \mathrm{U}: \operatorname{trn} \mathrm{L}(\mathrm{CUA}) ; \mathrm{V}: \operatorname{trnV}$. 


\section{Table notes}

702 Table 1 Species used to construct the phylogenetic relationships along with GenBank accession numbers. $703 *$ Partial genome.

704 Table 2. The partition schemes and best-fitting models selected of 13 protein-coding genes in the $13 \mathrm{P} 26$

705 dataset.

706 Table 3. The partition schemes and best-fitting models selected of 12 protein-coding genes in the $12 \mathrm{P} 38$

707 dataset.

708 Table 4 Base composition of Cerambycidae mitochondrial genomes.

709

710 Table S1 Universal and specific primers used to amplify the mitochondrial genomes of the five beetle species.

711 Table S2 Organization of the $O$. yaoshana mitochondrial genome.

712 Table $\mathbf{S 3}$ Organization of the $T$. croccocincta mitochondrial genome.

713 Table $\mathbf{S 4}$ Organization of the $B$. succinctor mitochondrial genome.

714 Table S5 Organization of the $N$. carinicollis mitochondrial genome.

715 Table S6 Organization of the Pterolophia sp. ZJY-2019 mitochondrial genome.

716 Table S7. The codon number and relative synonymous codon usage (RSCU) in mitochondrial protein coding 717 genes.

718

719 


\section{Figure 1}

Figure 1 The RSCU of five longicorn beetle mitochondrial genomes.

Figure 1 The RSCU of five longicorn beetle mitochondrial genomes. Codon families are provided on the $\mathrm{x}$-axis along with the different combinations of synonymous codons that code for that amino acid. RSCU (relative synonymous codon usage) is defined on the $\mathrm{Y}$ axis. 
O. yaoshana

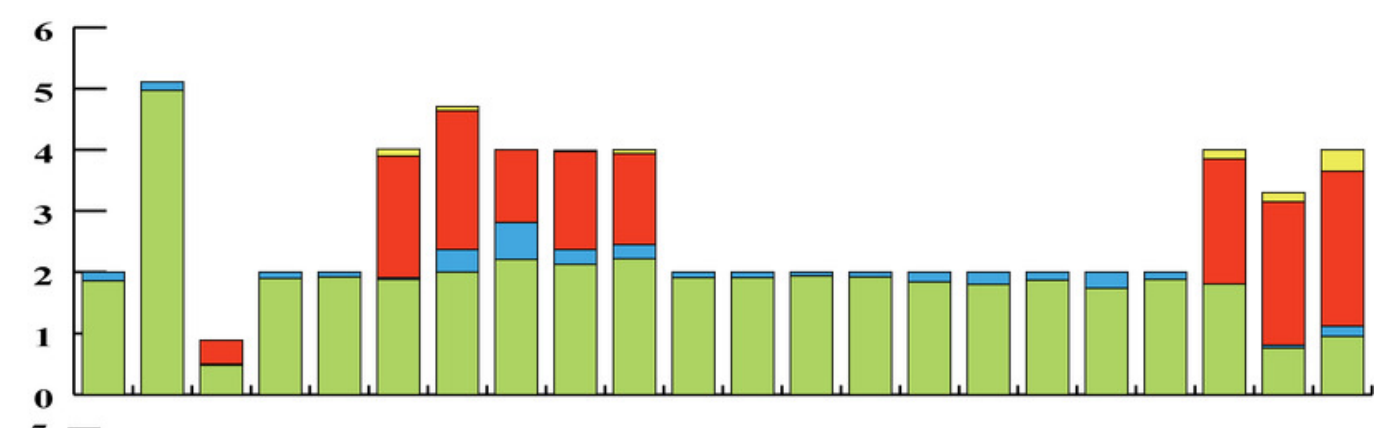

T. croccocincta

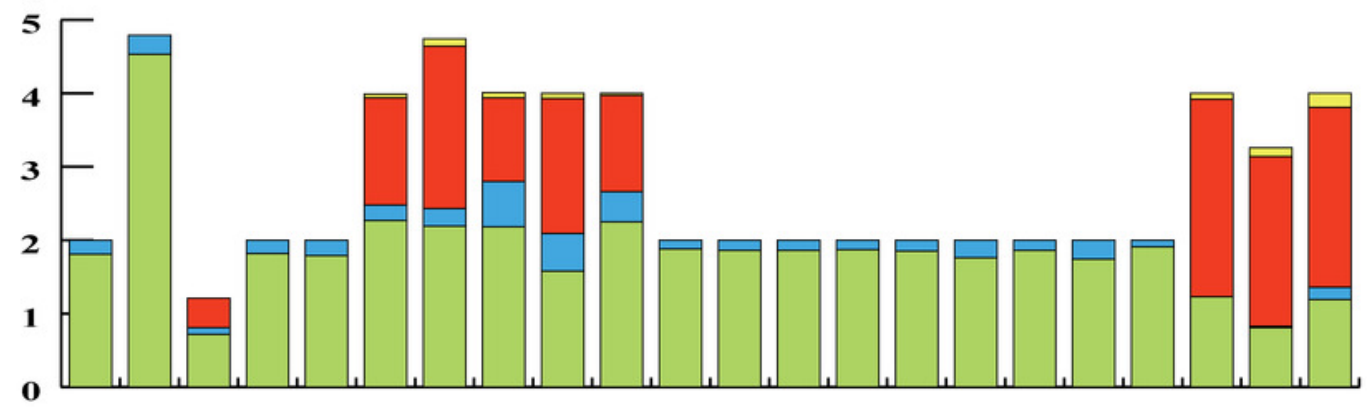

B. succinctor

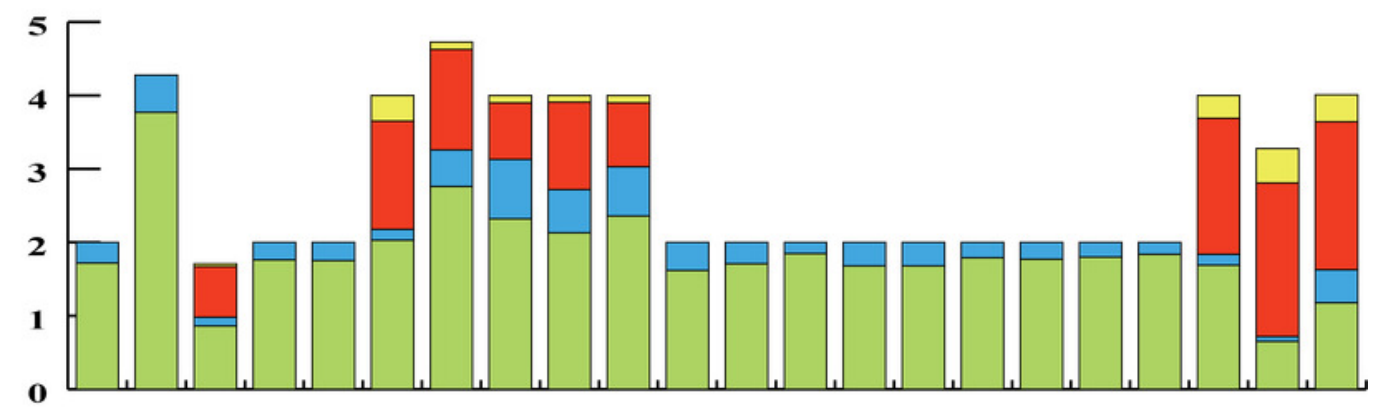

N. carinicollis

Pteropliini sp. ZJY-2019
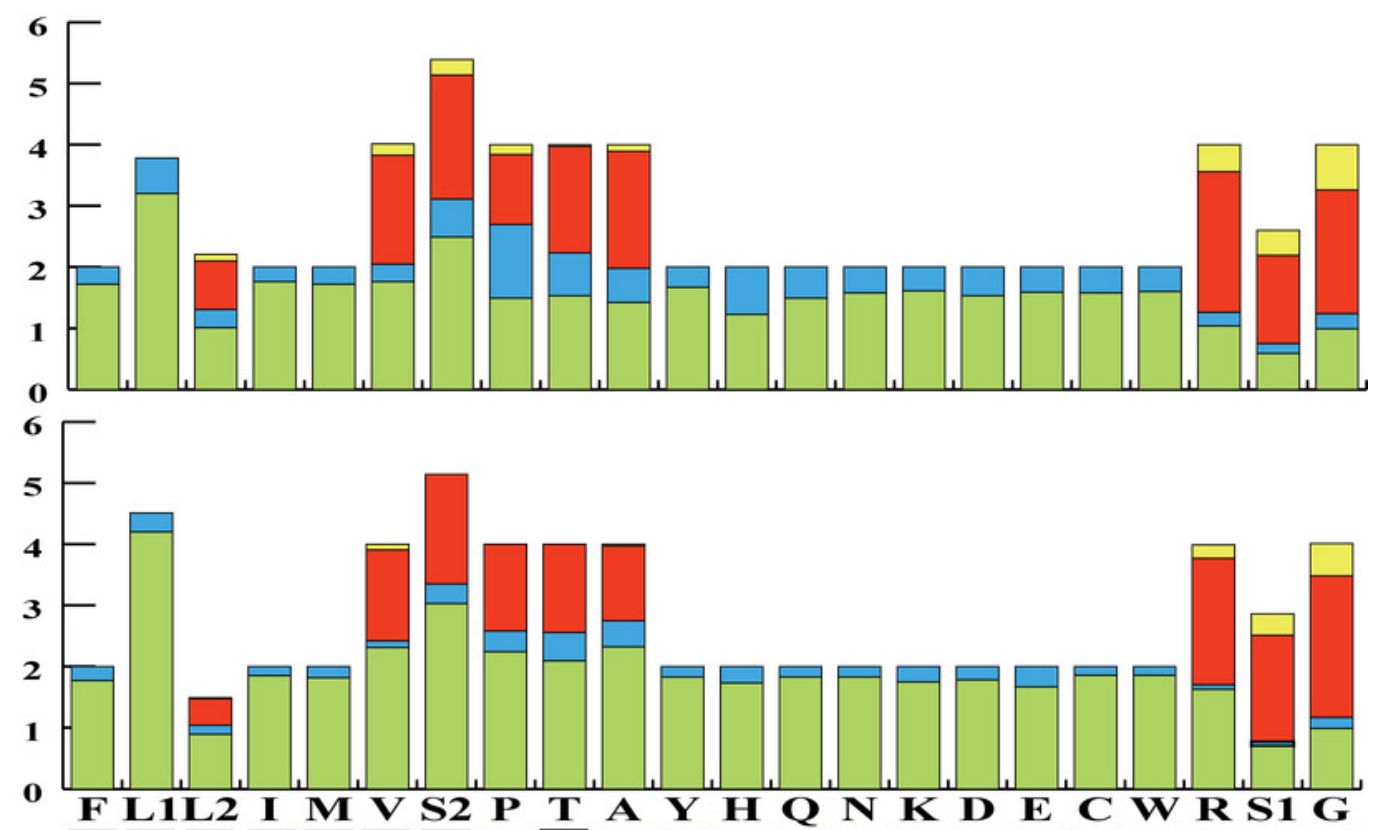

o F LIL2 I M V S2 P T A Y H Q N K D E C W R S1 G

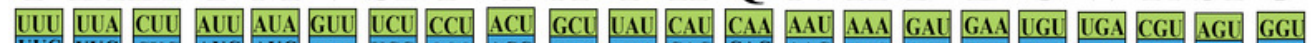
UUC UUG CUC AIC AUG GIC UCC CCC ACC GCC UAC CAC CAG AAC AAG GAC GAG UGC UGG CGC AGC GGC

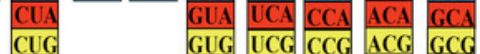




\section{Figure 2}

Figure 2 Putative mechanisms for formation of the two large intergenic regions (IGRs) that exist in Pterolophia sp. ZJY-2019.

Figure 2 Putative mechanisms for formation of the two large intergenic regions (IGRs) that exist in Pterolophia sp. ZJY-2019. (a) The slipped-strand mispairing and random loss model to explain the $157 \mathrm{bp}$-IGR between trnS2 and nad1. The CS indicates the 18 bp conservative sequence TTACTAAATTTAATTAACTAAA. (b) The duplication/random loss model to explain the $184 \mathrm{bp}-$ IGR between trnC and trnY.

$\mathbf{a}$
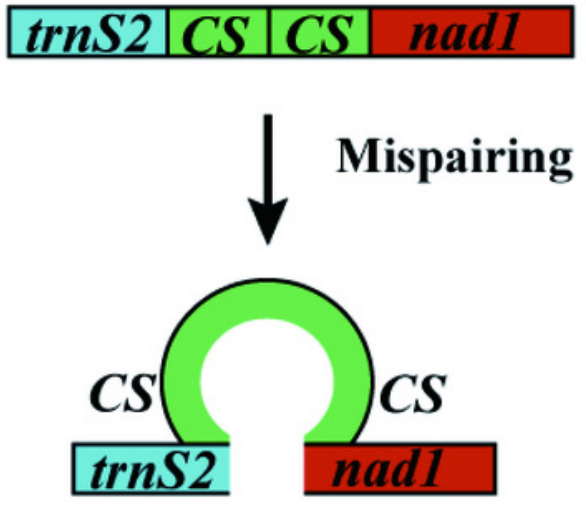

$\downarrow$ Expansion

\begin{tabular}{|l|l|l|}
\hline $\operatorname{trnS} 2$ & $\mathrm{CS}$ \\
\hline
\end{tabular}

$$
\downarrow \text { Random loss }
$$

b

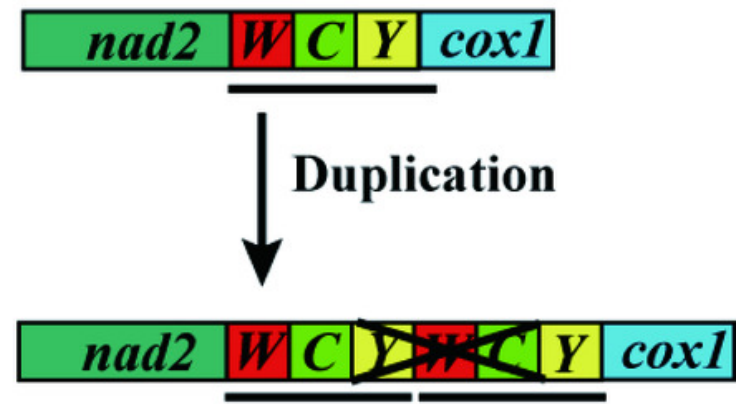

Random loss

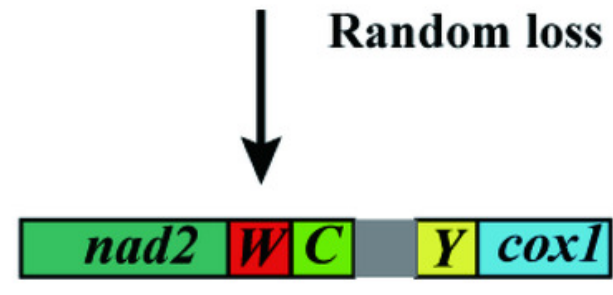

184 bp Intergenic region \begin{tabular}{|l|l|l|l|}
\hline trnS2 & $C S$ & $C S$ & nad1 \\
\hline
\end{tabular}

157 bp Intergenic region 
Figure 3

Figure 3 Phylogenetic relationships of Cerambycidae in $\mathrm{BI}$ and $\mathrm{ML}$ analyses.

Figure 3 Phylogenetic relationships of Cerambycidae in BI and ML analyses. The data includes 23 species of Cerambycidae as the ingroup and three species of Chrysomelidae as the outgroup. The GenBank accession numbers of all species are also shown.
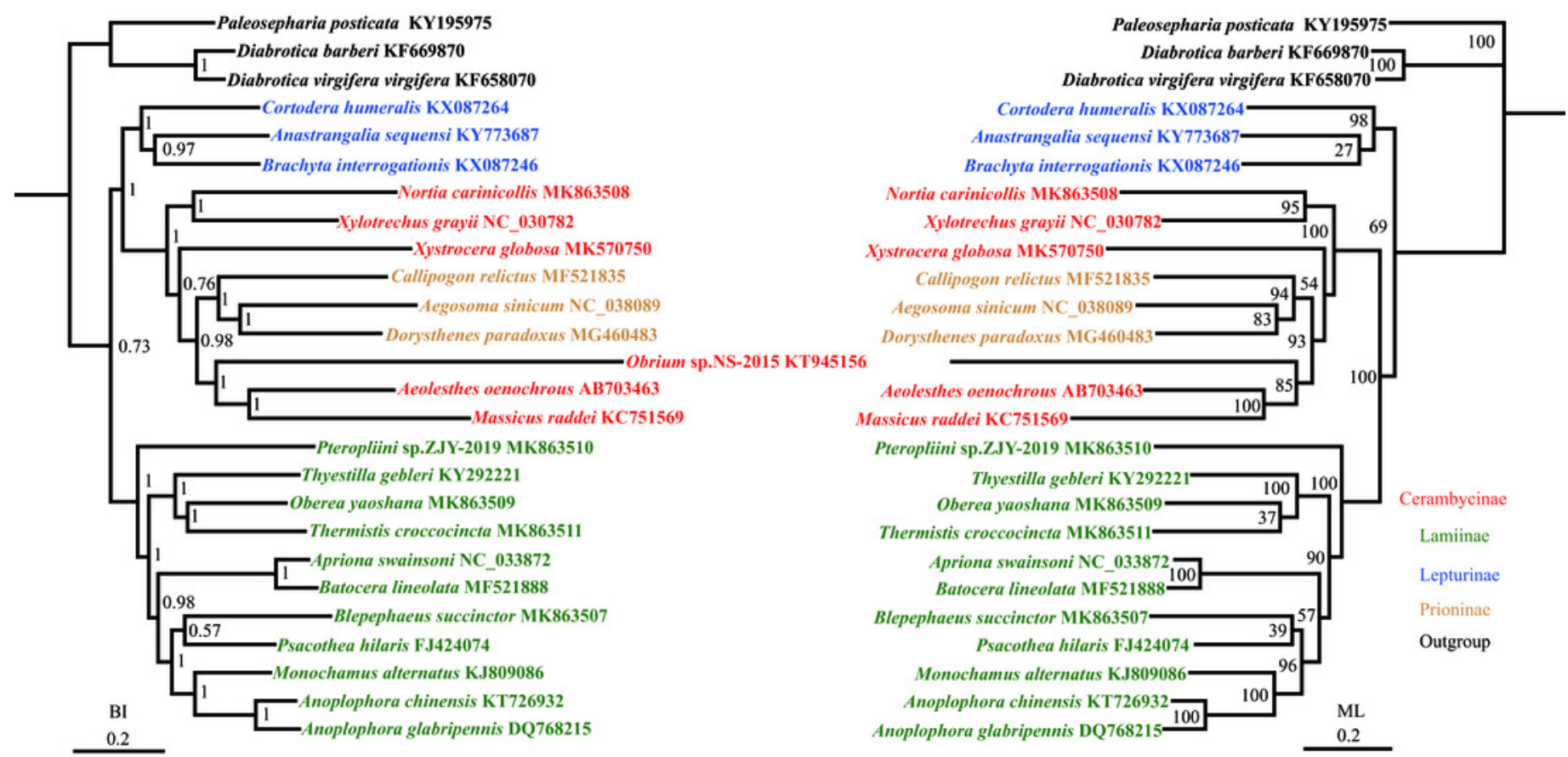
Figure 4

Figure 4 Phylogenetic relationships of Cerambycidae in $\mathrm{BI}$ and $\mathrm{ML}$ analyses.

Figure 4 Phylogenetic relationships of Cerambycidae in BI and ML analyses. The data includes 35 species of Cerambycidae as the ingroup and three species of Chrysomelidae as the outgroup. The GenBank accession numbers of all species are also shown.

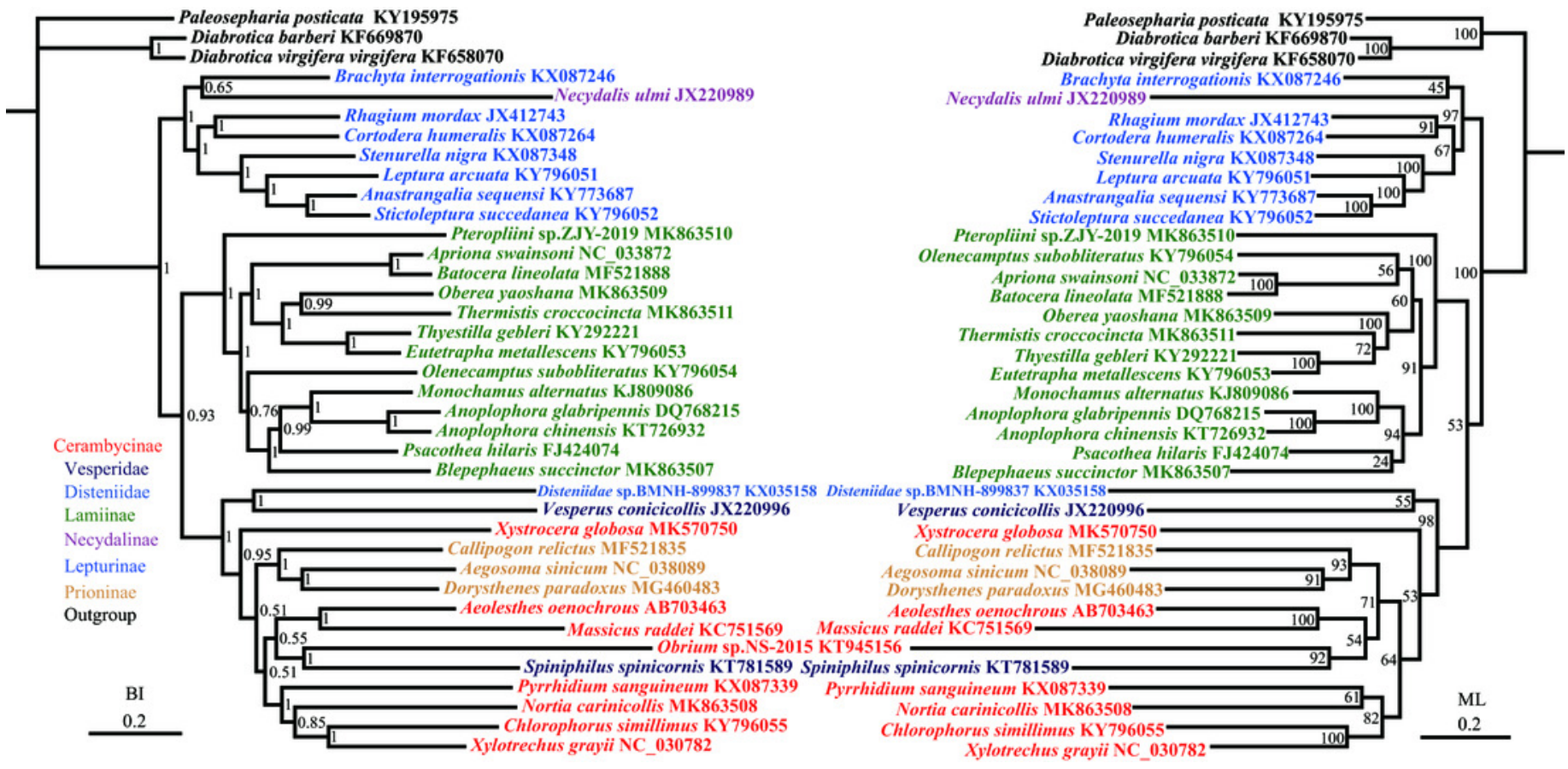




\section{Table $\mathbf{1}$ (on next page)}

Table 1 Species used to construct the phylogenetic relationships along with GenBank accession numbers. *Partial genome.

Table 1 Species used to construct the phylogenetic relationships along with GenBank accession numbers. *Partial genome. 


\begin{tabular}{|c|c|c|c|c|}
\hline Order & Family & Species & GenBank No. & References \\
\hline \multirow[t]{32}{*}{ Cerambycidae } & Lamiinae & Anoplophora glabripennis & DQ768215 & Fang et al., 2016 \\
\hline & & Psacothea hilaris & FJ424074 & Kim et al., 2009 \\
\hline & & Thyestilla gebleri & KY292221 & Yang et al., 2017 \\
\hline & & Monochamus alternatus & KJ809086 & Li et al., 2016a \\
\hline & & Anoplophora chinensis & KT726932 & Li et al., $2016 b$ \\
\hline & & Apriona swainsoni & NC_033872 & Que et al., 2019 \\
\hline & & Batocera lineolata & MF521888 & Liu et al., 2017 \\
\hline & & Oberea yaoshana & MK863509 & This study \\
\hline & & Thermistis croccocincta & MK863511 & This study \\
\hline & & Blepephaeus succinctor & MK863507 & This study \\
\hline & & Pterolophia sp.ZJY-2019 & MK863510 & This study \\
\hline & & Olenecamptus subobliteratus* & KY796054 & Directly submitted \\
\hline & & Eutetrapha metallescens* & KY796053 & Directly submitted \\
\hline & Cerambycinae & Xylotrechus grayii & NC_030782 & Guo et al., 2016 \\
\hline & & Xystrocera globosa & MK570750 & Wang et al., 2019 \\
\hline & & Nortia carinicollis & MK863508 & This study \\
\hline & & Massicus raddei & KC751569 & Wang et al., 2014 \\
\hline & & Aeolesthes oenochrous & $\mathrm{AB} 703463$ & Chiu et al., 2016 \\
\hline & & Obrium sp. NS-2015 & KT945156 & Song et al., 2017 \\
\hline & & Pyrrhidium sanguineum* & KX087339 & Directly submitted \\
\hline & & Chlorophorus simillimus* & KY796055 & Directly submitted \\
\hline & Prioninae & Callipogon relictus & MF521835 & Lim et al., 2017 \\
\hline & & Dorysthenes paradoxus & MG460483 & Liu et al., 2018 \\
\hline & & Aegosoma sinicum & NC_038089 & Directly submitted \\
\hline & Lepturinae & Leptura arcuata* & KY796051 & Directly submitted \\
\hline & & Stictoleptura succedanea* & KY796052 & Directly submitted \\
\hline & & Rhagium mordax* & JX412743 & Directly submitted \\
\hline & & Stenurella nigra* & KX087348 & Directly submitted \\
\hline & & Cortodera humeralis & KX087264 & Directly submitted \\
\hline & & Anastrangalia sequensi & KY773687 & Directly submitted \\
\hline & & Brachyta interrogationis & KX087246 & Directly submitted \\
\hline & Necydalinae & Necydalis ulmi* & JX220989 & Directly submitted \\
\hline Disteniidae & Disteniinae & Disteniinae sp. BMNH 899837 & KX035158 & Directly submitted \\
\hline \multirow[t]{2}{*}{ Vesperidae } & Philinae & Spiniphilus spinicornis & KT781589 & Nie et al., 2017 \\
\hline & Vesperinae & Vesperus conicicollis* & JX220996 & Directly submitted \\
\hline \multirow[t]{3}{*}{ Chrysomelidae } & Galerucinae & Paleosepharia posticata & KY195975 & Wang et al., 2017 \\
\hline & & Diabrotica barberi & KF669870 & Brad, 2014 \\
\hline & & Diabrotica virgifera & KF658070 & Brad, 2014 \\
\hline
\end{tabular}




\section{Table 2 (on next page)}

Table 2 . The partition schemes and best-fitting models selected of 13 protein-coding genes in the 13P26 dataset.

Table 2. The partition schemes and best-fitting models selected of 13 protein-coding genes in the 13P26 dataset. 
Table 2. The partition schemes and best-fitting models selected of 13 protein-coding genes in 13P26 data

\begin{tabular}{lll} 
& \multicolumn{2}{c}{ Nucleotide sequence alignments } \\
Subset & Subset Partitions & Best Model \\
\hline Partition 1 & atp6_pos1, cox1 pos 1, cox2_pos1, cox3_pos1, cytb_pos1 & GTR+I+G \\
Partition 2 & atp6_pos2, cox1_pos2, cox2_pos2, cox3_pos2, cytb_pos2, nd3_pos2 & TVM+I+G \\
Partition 3 & atp8_pos1, atp8_pos2, nd2_pos2, nd3_pos3, nd6_pos2 & GTR+I+G \\
Partition 4 & nd1_pos1, nd41_pos1, nd4_pos1, nd5 pos1 & GTR+I+G \\
Partition 5 & nd1_pos2, nd4_pos2, nd41_pos2, nd5_pos2 & GTR+I+G \\
Partition 6 & nd2_pos2, nd3_pos2, nd6_pos2 & TVM+I+G \\
\hline
\end{tabular}




\section{Table 3 (on next page)}

Table 3. The partition schemes and best-fitting models selected of 12 protein-coding genes in the $12 \mathrm{P} 38$ dataset.

Table 3. The partition schemes and best-fitting models selected of 12 protein-coding genes in the $12 \mathrm{P} 38$ dataset. 
1 Table 3. The partition schemes and best-fitting models selected of 12 protein-coding genes in $12 \mathrm{P} 38$ data.

\begin{tabular}{lll}
\hline & \multicolumn{1}{c}{ Nucleotide sequence alignments } & \\
Subset & Subset Partitions & Best Model \\
\hline Partition 1 & atp6_pos1, cox2_pos1, cox3_pos1, cytb_pos1 & GTR+I+G \\
Partition 2 & atp6_pos2, cox2_pos2, cox3_pos2, cytb_pos2, nd3_pos2 & TVM+I+G \\
Partition 3 & atp8_pos1, atp8_pos2, nd6_pos2 & HKY+G \\
Partition 4 & cox1 pos 1 & SYM+G \\
Partition 5 & cox1_pos2 & F81+G \\
Partition 6 & nd1_pos1, nd41_pos1, nd4_pos1, nd5 pos1 & GTR+I+G \\
Partition 7 & nd1_pos2, nd4_pos2, nd41_pos2, nd5_pos2, & GTR+I+G \\
Partition 8 & nd3_pos1, nd6_pos1 & GTR+I+G \\
\hline
\end{tabular}




\section{Table 4 (on next page)}

Table 4 Base composition of Cerambycidae mitochondrial genomes.

Table 4 Base composition of Cerambycidae mitochondrial genomes. 


\begin{tabular}{|c|c|c|c|c|c|c|c|c|c|c|c|c|}
\hline \multirow[t]{4}{*}{ Species } & \multicolumn{4}{|c|}{$\mathbf{A}+\mathbf{T}(\%)$} & \multicolumn{4}{|c|}{ AT-skew } & \multicolumn{4}{|c|}{ GC-skew } \\
\hline & \multirow[t]{3}{*}{ Mito } & \multirow[t]{3}{*}{ PCGs } & \multirow[t]{3}{*}{ rRNAs } & \multirow{3}{*}{$\begin{array}{l}\text { AT- } \\
\text { rich } \\
\text { region }\end{array}$} & \multirow[t]{3}{*}{ Mito } & \multirow[t]{3}{*}{ PCGs } & \multirow[t]{3}{*}{ rRNAs } & \multirow{3}{*}{$\begin{array}{l}\text { AT-rich } \\
\text { region }\end{array}$} & \multirow[t]{3}{*}{ Mito } & \multirow[t]{3}{*}{ PCGs } & \multirow[t]{3}{*}{ rRNAs } & \multirow{3}{*}{$\begin{array}{l}\text { AT-ricl } \\
\text { region }\end{array}$} \\
\hline & & & & & & & & & & & & \\
\hline & & & & & & & & & & & & \\
\hline O. yaoshana & 79.1 & 77.8 & 81.1 & 87.1 & 0.03 & 0.14 & 0.04 & 0.04 & 0.20 & 0.01 & 0.38 & 0.24 \\
\hline T. croccocincta & 76.4 & 76.4 & 78.6 & 87.4 & 0.15 & 0.15 & 0.04 & 0.04 & 0.13 & 0.01 & 0.49 & 0.45 \\
\hline B. succinctor & 75.3 & 73.2 & 78.6 & 86.2 & 0.023 & 0.17 & 0.06 & 0.02 & 0.26 & 0.02 & 0.39 & 0.32 \\
\hline N. carinicollis & 73.2 & 71.1 & 75.7 & 80.3 & 0.10 & 0.17 & 0.16 & 0.07 & 0.18 & 0.03 & 0.36 & 0.21 \\
\hline Pterolophia & 76.7 & 75.1 & 81.7 & 82.8 & 0.02 & 0.18 & 0.02 & 0.04 & 0.22 & 0.04 & 0.36 & 0.18 \\
\hline sp.ZJY-2019 & & & & & & & & & & & & \\
\hline
\end{tabular}

1 